\title{
Percepción de la experiencia de violencia doméstica en mujeres víctimas de maltrato de pareja*
}

\section{Perception of the Experience of Domestic Violence in Women Victims of Intimate Partner Violence}

Recibido: 19 de agosto de 2013 | Revisado: 5 de noviembre de 2014 | Aceptado: 13 de abril de 2015

\author{
JANNETH ElizabeTh Molina Rico ** \\ JAIME HuMBERTO MORENO MÉNDEZ
}

Universidad Católica de Colombia, Bogotá

doi:10.11144/Javeriana.upsy14-3.pevd

Para citar este artículo: Molina, J. E., \& Moreno, J. H. (2015). Percepción de la experiencia de violencia doméstica en mujeres víctimas de maltrato de pareja. Universitas Psychologica, 14(3), 997-1008. http://dx.doi.org/10.11144/Javeriana.upsy14-3.pevd

* Artículo de investigación

** Grupo de investigación. Facultad de Psicología. Universidad Católica de Colombia. Correos electrónicos: janmolina2003@yahoo.com.mx; jaimehm@yahoo.com. Auxiliares de investigación: Alexandra Bautista, Sonia Galvis, Patricia García, Claudia Giraldo, Sirley Olivarez, Adriana Penagos, Lizeth Rodríguez.

\section{RES U MEN}

El presente estudio tuvo como objetivo comprender las percepciones que sobre la violencia doméstica tiene un grupo de mujeres víctimas de la misma. Para tal fin, se llevó a cabo una investigación de tipo cualitativo bajo la técnica de grupos focales. Las participantes de la investigación fueron nueve mujeres víctimas de la violencia doméstica, con edades comprendidas entre los 25 y 60 años, atendidas en una ONG de la ciudad de Bogotá. Se encontró que las mujeres tienen una percepción negativa de sí mismas, aprendida desde sus familias de origen, pues minimizan muchas de sus capacidades y evalúan sus habilidades como inferiores a las de los hombres.

Palabras clave

violencia doméstica; percepciones; género

\section{A B S T R A C T}

This study aimed to understand the perceptions that domestic violence has a group of women victims of it. To this end we conducted a qualitative study on the technique of focus groups. The research participants were 9 women victims of domestic violence between the ages of 25 and 60 years served in an ONG of Bogotá city. It was found in women with a negative perception of themselves while minimizing many of the skills they possess, and evaluating their skills as inferior to men, which have learned from their families of origin.

Keywords

domestic violence; perceptions; gender 
La violencia doméstica es una problemática que genera consecuencias físicas y psicológicas que pueden llegar a ser fatales y que afectan no solo a sus víctimas sino a la sociedad en general. En el ámbito científico se encuentran diferentes aproximaciones conceptuales sobre este fenómeno que dan cuenta de la complejidad del mismo. Al respecto, Molina, Moreno y Vásquez (2010) refieren que la violencia doméstica tiene diferentes matices, y que en cuanto a su taxonomía no hay un consenso en la literatura, resaltando que los términos violencia intrafamiliar y violencia doméstica son manejados como diferentes por algunos autores y como sinónimos por otros.

Álvarez y Hartog (2005), Sanmartín, Molina y García (2003) y Lila (2010) la definen como cualquier tipo de violencia que tiene lugar en el seno de la familia, como un problema de derechos humanos y de salud pública que afecta a los individuos de la sociedad, resaltando que la violencia doméstica y la violencia familiar son términos muy similares. Garrido y Morales (2003) la definen como "toda forma de violencia psíquica, física o sexual perpetrada al interior de la familia" (p. 112). La violencia doméstica incluye todo acto violento en la pareja, ya sea de matrimonio, de convivencia o noviazgo, o aquellas relaciones ya disueltas por la separación.

En la literatura en inglés, Vatnar y Bjørkly (2014), O'Doherty, Taft, Hegarty, Ramsay y Feder (2014), Ellsberg y Heisse (2005), Lilly y Graham-Bermann (2010), Saftlas et al. (2014) y Singh, Petersen y Singh (2014) emplean el término 'Intimate partner violence (IPV)', para referirse a la violencia doméstica; sin embargo, en español se continúan utilizando los términos violencia doméstica, conyugal o de pareja. Labrador, Paz, De Luis y Fernández-Velasco (2008) utilizan el término la violencia doméstica para hacer referencia a "la violencia conyugal y dentro de ésta a aquellos casos, la gran mayoría, en que la víctima es la mujer" (p. 24).

Por otra parte, Walker (1999) propone como concepto de la violencia doméstica un patrón de conductas abusivas que incluye un amplio rango de maltrato, físico, sexual y psicológico, usado por una persona en una relación íntima contra otra, para ganar poder o para mantener el abuso de poder, control y autoridad sobre esa persona. Esta definción es la que se toma en este estudio, circunscribiéndola al maltrato del que es víctima la mujer por parte de su pareja.

Varios autores proponen diferentes tipos de maltrato, entre ellos, el maltrato fisico, psicológico, sexual, emocional, verbal, económico y social (Carrasco, 2007; Gage, 2005; Labrador et al., 2008; Organización Panamericana de la Salud [OPS], 2003; Zolotor, Denham, \& Weil, 2009). La violencia física se refiere a todo acto de agresión intencional en que se emplea alguna parte del cuerpo de la mujer, algún objeto, arma o sustancia para sujetar, inmovilizar o causar daño o intento de daño, permanente o temporal, de parte del agresor sobre el cuerpo de ella (Instituto Nacional de Mujeres, 2006; Fondo Internacional de Emergencia de las Naciones Unidas para la Infancia, 2000).

La violencia psicológica hace referencia a las múltiples actitudes y conductas que una persona tiene hacia su pareja en cuanto a denigrar, criticar, humillar, intimidar, amenazar, controlar, sobrerresponsabilizar, simular indiferencia y minimizar situaciones (Álvarez \& Hartog, 2005). Según la World Health Organization ([WHO], 2005), una forma de violencia psicológica es la violencia social, la cual se caracteriza por que el agresor limita los contactos tanto sociales como familiares de su pareja, lo que se traduce en el aislamiento social y la disminución de su círculo de apoyo. Según Álvarez y Hartog (2005) y Labrador et al. (2008), la violencia sexual implica "cualquier intimidad sexual forzada por parte de la pareja, ya sea con amenazas, intimidación, coacción o por llevarse a cabo en estado de inconsciencia o indefensión de la mujer" (p. 26).

En la última década, varias encuestas a mujeres con algún tipo de unión conyugal, han establecido que entre el $33 \%$ y el $37 \%$ ha sufrido algún tipo de violencia verbal y entre un $19.3 \%$ y un $39.5 \%$, violencia física (Banco Mundial, 2002; Organización Mundial de la Salud [OMS], 2005). En un informe elaborado por la Organización Mundial de la Salud, Escuela de Higiene y Medicina Tropical de Londres y el Consejo Sudafricano de Investigaciones Médicas (2013), se encontró que el 35 \% de las mujeres ha sido víctima de violencia física y sexual por parte de su pareja. 
En Colombia, López, Murad y Calderón (2013) encontraron que el $74.6 \%$ de mujeres han sido violentadas por su pareja, en el último año. Según el Instituto Nacional de Medicina Legal y Ciencias Forenses de Colombia ([INML], 2013), durante el 2013 el $87.21 \%$ de las víctimas valoradas por maltrato, fueron las mujeres.

En relación con los factores de riesgo de la violencia doméstica, el Informe Mundial sobre la Violencia y la Salud de la OPS y la OMS (2003) y el Fondo de Desarrollo de las Naciones Unidas para la Mujer (UNIFEM), Fondo de Población de las Naciones Unidas (UNFPA), Organización Internacional para las Migraciones (OIM) (2010), plantean que el maltrato a la mujer sucede en las sociedades en las que existe rigidez en los roles de género, normas culturales que respaldan el derecho del hombre a mantener relaciones sexuales con independencia de los sentimientos de la mujer y sanciones blandas para estos comportamientos.

Para Labrador et al. (2008) "las conductas violentas son conductas aprendidas a partir de modelos familiares y sociales que consideran la violencia como un recurso válido para resolver conflictos" (p. 34). Echeburúa y De Corral (2009) consideran, dentro de los principales factores de riesgo para esta problemática, los siguientes:

Estructura familiar autoritaria y verticalista, aprendizaje de roles de género estereotipados, modelos de resolución violenta de conflictos en la familia de orígen, ausencia de modelos sociales que actúen como reforzadores negativos de la violencia, pautas culturales legitimadoras de la violencia y respuestas institucionales y comunitarias inadecuadas para los casos identificados. (p. 178)

En relación con los factores culturales y de género, los imaginarios, los prejuicios y las representaciones sociales se pueden constituir en factores de riesgo de las relaciones violentas en la pareja, como los develados en un estudio por Molina et al. (2010), tales como que "el hombre es superior a la mujer, la mujer nace para ser esposa y madre, tolerar la violencia es una cuestión de principios, la mujer que obedece a su marido no es víctima de violencia” (p. 135), entre otros, los cuales se pueden entender como dispositivos de control y poder, que facilitan y perpetúan la violencia contra la mujer y el desempoderamiento del cual ella es víctima.

Para dar cuenta de la interacción, la multiplicidad y diversidad de factores que pueden determinar la aparición de la violencia doméstica, una de las herramientas conceptuales de mayor aceptación y uso es el Modelo Ecológico, basado en la propuesta original de Bronfenbrenner (1979) y utilizado por primera vez por Lori Heisen (1998 como se citó en López et al., 2013) y posteriormente por un gran número de autores (Bosh, Ferrer, \& Alzamora, 2006; López et al., 2013; Incháustegui \& Olivares, 2011; OMS, 2005). Este modelo integra todos los factores de riesgo en cuatro ámbitos: individual, familiar, comunitario y el social (Bosh et al., 2006).

En cuanto a las consecuencias, la violencia doméstica puede conducir a diversas formas de morbilidad y mortalidad a través de vías directas e indirectas, lo que configura un amplio rango de efectos sobre la salud de la mujer (OMS, 2013). Según Llorente (2001 como se citó en Labrador, Arinero, \& Crespo, 2004) "Los malos tratos son la tercera causa que está provocando más muertes prematuras y más secuelas físicas y psíquicas en las mujeres" (p. 61). Dentro de las consecuencias físicas, se encuentran lesiones (musculoesqueléticas, de tejido blando, trauma genital), síndrome de dolor crónico, trastornos gastrointestinales, daño ocular, reducción del funcionamiento físico y discapacidad. En relación con las consecuencias conductuales y psicológicas, se encuentran trastorno de estrés postraumático, trastornos de ansiedad, depresión, trastornos alimentarios y del sueño, trastornos psicosomáticos, baja autoestima, conducta sexual insegura, tabaquismo, abuso de alcohol y drogas, conducta suicida y autodestructiva. Como consecuencias a nivel sexual y reproductivo, se encuentran: trastornos ginecológicos, infertilidad, embarazos no deseados, complicaciones en el embarazo y aborto, disfunciones sexuales y enfermedades de transmisión sexual. Como consecuencias fatales, mortalidad relacionada con 
el SIDA, mortalidad materna, homicidio y suicidio (Glantz, Halperin, \& Hunt, 2000; Labrador et al., 2008; Sanmartín et al., 2003; OMS, 2003; OPS, 2013). La principal consecuencia de este problema de acuerdo con la UNICEF (2000) es la negación de los derechos fundamentales. En un estudio realizado por Hindin, Kishor y Ansara (2008), se encontró que la mujer que ha experimentado violencia doméstica tiene una probabilidad más alta de tener embarazos que terminan en mortalidad natal.

En Colombia en un estudio realizado por Profamilia, se encontró que el porcentaje de mujeres que sufrieron lesiones físicas en el 2005 fue de 71 \% y en el 2010 de 72 \% (Murad, Gómez, \& Caderón, 2013). Las lesiones emocionales como consecuencia de la violencia se presentan en el $80 \%$ de los casos, y se hacen más frecuentes después de los 24 años.

Para avanzar en la comprensión de la violencia doméstica, es importante hacer una aproximación a la dinámica que la perpetúa, la cual queda plasmada en el llamado ciclo de la violencia propuesto por Walker $(1979 ; 1989)$, el cual se presenta bajo tres fases, la primera, la Acumulación de tensión, que hace referencia a episodios menores de maltrato,en donde por lo general se dan agresiones psicológicas que son ignoradas por la víctima. En la segunda fase, denominada Explosión o agresión, el agresor pierde el control y ejerce violencia fisica, sexual o psicológica ocasionando graves consecuencias para la víctima. La tercera fase, llamada Reconciliación o luna de miel, se caracteriza por el arrepentimiento del victimario, quien pide perdón y se compromete a cambiar. Estas fases cada vez se hacen más cortas y en ocasiones desaparece la fase de reconciliciación, pasando de la tensión a la agresión lo cual agrava la situación (Walker, 1979; 1989; 2012). El entender por qué un gran número de mujeres no denuncia y continúa en una relación violenta, requiere conocer y comprender cuáles son las estrategias de afrontamiento que utilizan las víctimas y sobrevivientes de la violencia doméstica.

El afrontamiento ha sido conceptualizado por Lazarus y Folkman (1984) como "aquellos esfuerzos cognitivos y conductuales cambiantes que se desarrollan para manejar las demandas específicas externas y/o internas que son evaluadas como excedentes o desbordantes de los recursos del individuo" (p. 164). Rodríguez (1995) y Kleinke (2001) definen los recursos de afrontamiento como elementos y/o capacidades, internos o externos, con los que cuenta la persona para enfrentar a las demandas del medio potencialmente dañinas o estresantes.

Existen diversas clasificaciones de las estrategias de afrontamiento. Lazarus y Folkman (1988) y Lazarus (2000) las clasifican en manejo confrontador, distanciamiento, autocontrol, búsqueda de apoyo social, aceptación de la responsabilidad, escape-evitación, resolución de problemas y revaloración positiva. Rodríguez (1995) propone una taxonomía según el foco (orientadas a la emoción, el problema, la situación, la representación o la evaluación), según el método (aproximativas, pasivas y evitativas), según el tipo de proceso (comportamentales y cognitivas), según el momento (anticipatorias y restaurativas) y según la amplitud (globales y específicas). Lazarus (2000) y Lazarus y Folkman (1988) proponen una clasificación que tiene como referente la función e incluye dos tipos de estrategias de afrontamiento, el centrado en el problema y el centrado en la emoción. Una de las clasificaciones más conocidas y utilizadas es la de las estrategias de afrontamiento de aproximación y de evitación (Moos, 1995 citado por Krause, Kaltman, Goodman, \& Dutton, 2008).

Flanagan, Jaquier, Overstreet, Swan y Sullivan (2014) afirman que existe una asociación entre la estrategia de afrontamiento de evitación y resultados negativos en la salud mental de mujeres que han sufrido violencia de pareja o doméstica. También se ha hallado que la estrategia de evitación media la relación entre la violencia de pareja y la severidad de los síntomas de estrés postraumático, de depresión y en problemas de uso de sustancias en las mujeres víctimas de dicha violencia. Krause et al. (2008) también han encontrado una asociación entre afrontamiento centrado en la emoción (incluida la evitación) y mayor sintomatología de estrés postraumático en mujeres sobrevivientes de la 
violencia de pareja. Flanagan et al. (2014) reportan que la estrategia de afrontamiento de evitación como respuesta a la violencia de pareja, tiene efectos negativos a largo plazo, y que dicha estrategia también puede funcionar como un método de normalización y regulación para reducir la angustia asociada a la violencia de pareja.

Hayati, Erikson, Hakimi, Högberg y Emmelin (2013) proponen la estrategia de afrontamiento denominada "banda elástica" para dar cuenta de la tensión y conflicto que experimenta la mujer entre escapar o permanecer en la relación violenta de pareja y los efectos psicosociales que arruinan su vida. Una de las formas de avanzar en la comprensión de este tema es abordarlo desde la percepción de las vivencias y experiencias de sus víctimas, para lo que los grupos focales, técnica elegida para esta investigación, constituyen una de las estrategias recomendadas en la medida en que, como lo plantea Davis (2002), a través de ella, una mujer sobreviviente de la violencia es capaz de formular su historia desde el pasado hasta el presente, y puede, junto con otras mujeres, explorar, compartir y validar sus sentimientos y puntos de vista. En este sentido, el objetivo de la presente investigación es comprender las percepciones que sobre la experiencia de violencia doméstica tiene un grupo de mujeres víctimas de la misma.

\section{Método}

\section{Participantes}

Las participantes en la investigación fueron mujeres víctimas de la violencia doméstica, atendidas en una ONG de la ciudad de Bogotá; no hubo ningún criterio de exclusión. El grupo total quedó constituido por 9 mujeres, distribuidas en 2 grupos focales con edades comprendidas entre los 27 y 60 años, los datos de todas las participantes se presentan en la Tabla 1.

\section{Técnica}

La entrevista a grupos focales es una técnica para la recolección de datos cualitativos en corto tiempo y en profundidad. Se realiza "a partir de una discusión con un grupo de seis a doce personas, quienes son guiadas por un entrevistador para exponer sus conocimientos y opiniones sobre temas considerados importantes para el estudio" (Bonilla \& Rodríguez, 1995, p. 194). Esta entrevista se caracteriza por ser uno de los instrumentos con procedimientos de evaluación rápida, que permiten a su vez comprender las actitudes, el saber cultural, las creencias y las percepciones de la comunidad en relación con la problemática que se investiga. Así mismo, la entrevista de grupos focales

TABLA 1

Características sociodemográficas de las participantes

\begin{tabular}{|c|c|c|c|c|c|c|c|c|c|}
\hline $\begin{array}{c}\text { Edad } \\
\text { (En años) }\end{array}$ & 41 & 50 & 32 & 31 & 28 & 27 & 43 & 60 & 46 \\
\hline $\begin{array}{c}\text { Ciudad de } \\
\text { origen }\end{array}$ & Bogotá & Bogotá & $\begin{array}{l}\text { Campo- } \\
\text { alegre } \\
\text { (Huila) }\end{array}$ & Bogotá & $\begin{array}{c}\text { Paipa } \\
\text { (Boyacá) }\end{array}$ & Bogotá & Bogotá & $\begin{array}{l}\text { Jenesano } \\
\text { (Boyacá) }\end{array}$ & Bogotá \\
\hline Estrato & 4 & 3 & 2 & 2 & 1 & 2 & 3 & 2 & 3 \\
\hline $\begin{array}{c}\text { Estado } \\
\text { civil }\end{array}$ & unión libre & casada & soltera & soltera & divorciada & unión libre & casada & casada & divorciada \\
\hline $\begin{array}{l}\text { Nivel } \\
\text { escolar }\end{array}$ & $\begin{array}{l}\text { Técnico } \\
\text { medio }\end{array}$ & $\begin{array}{c}\text { Secundaria } \\
\text { completa }\end{array}$ & $\begin{array}{l}\text { Técnico } \\
\text { superior }\end{array}$ & $\begin{array}{c}\text { Secundaria } \\
\text { completa }\end{array}$ & $\begin{array}{c}\text { Secundaria } \\
\text { completa }\end{array}$ & $\begin{array}{l}\text { Secundaria } \\
\text { incompleta }\end{array}$ & $\begin{array}{l}\text { Secundaria } \\
\text { incompleta }\end{array}$ & $\begin{array}{c}\text { Primaria } \\
\text { incompleta }\end{array}$ & $\begin{array}{l}\text { Técnico } \\
\text { medio }\end{array}$ \\
\hline Trabajo & Si (Pintora) & $\begin{array}{l}\text { Si (Ases. } \\
\text { Comerc) }\end{array}$ & No & $\begin{array}{c}\text { Si (Aux. } \\
\text { textil) }\end{array}$ & $\begin{array}{l}\text { No (Hogar, } \\
\text { estudia) }\end{array}$ & $\begin{array}{l}\text { Si. Diseños } \\
\text { y acabados } \\
\text { en cuero }\end{array}$ & $\begin{array}{c}\mathrm{Si} \\
\text { (Vendedora) }\end{array}$ & No (Hogar) & $\begin{array}{l}\text { Sí (Aux. } \\
\text { enferm.) }\end{array}$ \\
\hline $\begin{array}{l}\text { Familiares } \\
\text { con quien } \\
\text { convive }\end{array}$ & $\begin{array}{l}\text { Esposo, hija, } \\
\text { esposa }\end{array}$ & $\begin{array}{c}\text { Esposo y } \\
\text { esposa }\end{array}$ & $\begin{array}{c}\text { Mamá y } 4 \\
\text { hijos }\end{array}$ & $\begin{array}{l}\text { Mamá y dos } \\
\text { hijos (1 mes } \\
\text { y } 11 \text { años) }\end{array}$ & $\begin{array}{l}\text { Mamá y tres } \\
\text { hijos (12, } 6 \text { y } \\
3 \text { años) }\end{array}$ & $\begin{array}{c}\text { Esposo, } \\
\text { esposa y tres } \\
\text { hijos }\end{array}$ & No contestó & No contestó & $\begin{array}{c}\text { Exesposo } \\
\text { Hijos (18 y } \\
13 \text { años) }\end{array}$ \\
\hline
\end{tabular}

Fuente: elaboración propia 
consiste en identificar esos elementos, el conocimiento y la experiencia que son específicos de cada grupo y contrastarlos con los de otro, buscando semejanzas y diferencias. Carey (2003) plantea que con una idea adecuada del líder del grupo focal, los miembros pueden describir abundantes detalles de las experiencias complejas y los razonamientos que impulsan sus acciones, creencias, percepciones y actitudes; para el caso particular del presente estudio, los relacionados con la violencia doméstica.

\section{Procedimiento}

Se llevó a cabo a partir de la propuesta de Carey (2003), en tres fases: de preparación, de implementación y de análisis a interpretación de la información.

\section{Fase de preparación}

Se establecieron dos categorías orientadoras: tipos de maltrato y estrategias de afrontamiento. Se elaboró una guía de entrevista, que incluyó preguntas sobre los tópicos de interés, las cuales se manejaron de manera flexible. Se solicitó y obtuvo la autorización de la institución. Se hizo contacto con las participantes y como contraprestación para las mismas y para la institución se ofreció un Taller de Fortalecimiento de la Autoestima. Se hizo un entrenamiento de tres sesiones con todo el grupo de investigación y se seleccionaron dos investigadoras para liderar los grupos focales.

\section{Fase de implementación}

Los grupos focales se realizaron según la agenda programada, previa firma del consentimiento informado, garantizando la confidencialidad de la información. Su duración fue de dos horas. Las sesiones fueron grabadas, previa autorización de las participantes.

\section{Fase de análisis a interpretación de la información}

Se hizo la transcripción de las entrevistas, se elaboraron matrices para categorizar la información, se contrastaron los datos de las transcripciones y se integró la información de los dos grupos focales.

\section{Análisis e interpretación de la información}

En el presente estudio, se ha buscado ir a la percepción misma de las mujeres que se han visto directamente afectadas por la violencia doméstica en su contexto local, mediante el análisis de sus propios relatos con el fin de tener un diagnóstico que sirva de insumo para diseñar un programa de prevención sobre esta problemática. A continuación, se realiza una descripción y la interpretación de los aspectos más significativos, a partir de las categorías orientadoras y emergentes: tipos de violencia domésti$\mathrm{ca}$, redes de apoyo, estrategias de afrontamiento y consecuencias del maltrato.

\section{Tipos de maltrato}

(...) en las comidas me decía que yo era la más fea y vieja de todas; un día iba borracho me cogió a la fuerza me violó por el recto, yo lo único que hacía era cubrirme el estómago para que mi bebé no se fuera a morir. (María)

(...) las amenazas con mi esposo, llegaron al punto que me decían que él estaba pagando 4’000.000 de pesos para que me mataran. (Ana)

En los anteriores testimonios se identifica un patrón de maltrato variado de tipo físico, sexual, psicológico y económico, y de diversa intensidad y gravedad, generando indefensión en la mujer. Lo anterior coincide con lo planteado por Walker (1999), Garrido y Morales (2003) y Álvarez y Hartog (2005) quienes consideran la violencia doméstica como un patrón abusivo usado por una persona en una relación de poder íntima contra otra.

\section{Redes de apoyo}

La falta de redes de apoyo se constituye en factor de riesgo y de mantenimiento de las relaciones violentas, por cuanto genera en las mujeres un 
sentimiento de indefensión y vulnerabilidad frente al maltrato. Es importante diferenciar dos tipos de redes en estos relatos: unas naturales, circunscritas a familiares y amigos, y otras institucionales, referidas a las comisarías y profesionales que atienden las solicitudes de apoyo de estas mujeres. En relación con las primeras, las de familiares y amigos, se encontraron los siguientes relatos:

(...) yo sufría sola sola, menos mal encontré a una persona cerca a mí porque yo no tenía familia, no tenía a quien comentárselo y yo aguanté esas cosas solita entonces debido a tanto aguante eso sale y yo viví muy triste. (Blanca)

(...) yo tengo a mi mami que se vino a vivir un año acá a Bogotá pero ella está allá y yo acá, y ese es mi problema y yo mis problemas los resuelvo yo sola y lo acepto. (Sandra)

Se observa que, en algunos casos, las mujeres se encuentran realmente solas, ya que no tienen familiares o amigos a los cuales recurrir; en otros, cuando cuentan con algunos familiares, no acuden a ellos por vergüenza, por considerar que "la ropa sucia se debe lavar en casa" o porque creen que no pueden recibir la respuesta oportuna y adecuada para enfrentar el maltrato. Vale la pena resaltar que una de las formas que el hombre utiliza para ejercer su poder, es aislando a las mujeres, es decir, impidiendo que tengan las mencionadas redes de apoyo. En los relatos, se observa cómo el hecho de que una de las participantes haya podido contar con el apoyo de sus familiares, fue decisivo para que ella lograra salir de la relación abusiva. En relación con el segundo tipo de redes, las profesionales e institucionales, se encuentran el siguiente discurso:

Cuando mis gemelas tenían 6 meses de nacidas mi esposo les pegó y fue la primera vez que fui a la Comisaría a demandarlo por maltrato a menores, desde entonces ya perdí la cuenta de las veces que he ido a la Comisaria y desde entonces siempre es la boleta, y si no va, no pasa nada. (Paola)
Pues ya llega un límite que ya uno no soporta y tiene que buscar ayuda, por eso es que va uno a donde el psicólogo o va uno a la Comisaría, porque es que uno ya necesita buscar una ayuda porque uno ya no sabe que, qué hacer, yo fui a la Comisaría pero como todas dicen, eso no sirve pànada. (Carolina)

Las mujeres reportan que acuden solo en situaciones extremas a instituciones como comisarias, Policía o Bienestar Familiar, que aunque en algunos casos les dan a conocer sus derechos, perciben que no encuentran la protección y apoyo que requieren, por lo que se sienten re-victimizadas. Esta situación se constituye en otra de las razones por las cuales las mujeres optan por no volver a denunciar, agregando una mayor dosis de desamparo e indefensión a su problemática.

\section{Estrategias de afrontamiento}

(...) y muchas veces o sea tratar de hacer lo que el pidiera, o sea, venga para acá, siéntese acá, sírvame la comida, haga esto, haga lo otro. (Paola)

Mejor dicho casi ni quejarme para, si, como por llevar uno ahí delante de la gente el qué dirán que uno no quiso un hogar, pero no, yo creo que de verdad uno no merece eso. El cuento de la mamá, pues imagínese yo tengo 50 años cuántos años cree que tiene mi mamá, entonces la mamá de uno no es, que no, no se vaya a separar, que no se qué, que eso es para, que vea yo con su papá cómo he vivido. (Teresa)

En los discursos de las participantes se observa que las mujeres emplean diferentes formas para enfrentar el maltrato de sus parejas; en algunos casos, optan por ceder a sus peticiones con el fin de evitar ser agredidas lo que refuerza el rol autoritario del hombre y aumenta el grado de control que este ejerce sobre la mujer. Otras mujeres, refieren llanto, agresividad y aislamiento, tratando de evitar ser juzgadas por el medio familiar y social. En otros casos, hay quienes asumen la situación autoinculpándose con el fin de normalizar lo que les está pasando; por otro lado, hay quienes deciden separarse y seguir adelante con su vida. 
De acuerdo con la perspectiva del aprendizaje social, haber observado los roles del agresor y de la víctima como opción para solucionar problemas, es decir, del manejo del poder en las relaciones de pareja y el tipo de estrategias que se pueden y deben usar para enfrentar el maltrato, conlleva a un aprendizaje que es replicado generación tras generación (Labrador et al., 2008). Echeburúa y Del Corral (2009) consideran, dentro de los principales factores de riesgo para esta problemática, la estructura familiar autoritaria y verticalista, el aprendizaje de roles de género estereotipados, modelos de resolución violenta de conflictos en la familia de orígen, pautas culturales legitimadoras de la violencia y respuestas institucionales y comunitarias inadecuadas para los casos identificados.

La importancia de los tipos de estrategias de afrontamiento y la necesidad de profundizar en ellas, queda ilustrada por Lilly y Graham-Bermann (2010), quienes encontraron que las mujeres que han experimentado violencia doméstica y han empleado el afrontamiento centrado en la emoción, tienen mayor riesgo de desarrollar síntomas de estrés postraumático. Asimismo, Pineles et al. (2011) plantean que los individuos relativamente muy dependientes de las estrategias de afrontamiento de evitación y relativamente muy reactivos a los recuerdos del trauma pueden estar en mayor riesgo de mantener o incrementar potencialmente los síntomas de estrés postraumático en los primeros meses después del trauma.

\section{Consecuencias}

A mí me cortó la sonrisa, a mi me gustaba bailar, me gustaban muchas cosas, me hubiese gustado hacer muchas cosas pero me sentí amarrada porque permití muchas cosas. (María)

Él me ha contaminado tres veces con la Gardenella con hongos y la última vez me contaminó con el virus del Papiloma Humano. (María)

Después yo lo voy a tratar mal o él me va a tratar mal, peor de cómo nos hemos tratado ya, isi?, que no nos hemos respetado, que como lo digo yo, no tenemos dignidad. (Teresa)

En cuanto a las consecuencias, resultado del maltrato que se evidencian en los relatos anteriores, se identifican tres clases: a nivel físico, psicológico y social. Al respecto, Fernández et al. (2003) plantean, frente a las consecuencias del maltrato físico, que las víctimas evidencian lesiones, como hematomas, traumatismos, ITS y abortos. La consecuencia más grave es la muerte, la cual constituye, el costo más alto que puede cobrar la violencia doméstica.

En cuanto a las psicológicas, hay presencia de emociones de tristeza y ansiedad, desconfianza en sí mismas y hacia su pareja, temor a malos tratos, temor al futuro, en otras palabras, se presentan desesperanza. En varios casos, los esposos de estas mujeres tenían relaciones sentimentales paralelas con mujeres más jóvenes, con las cuales compartían más tiempo, y muchas veces se olvidaban de llegar a la casa; en sus relatos, las participantes manifiestan que en distintas oportunidades llegaron a hacerles saber todo lo que hacían en la intimidad y a compararlas con sus amantes.

La desesperanza aparece también en las ocasiones en que las víctimas deciden darle una oportunidad a sus esposos para mejorar la relación; sin embargo, pasado un tiempo, ellos vuelven a maltratarlas, y estas empiezan a resignarse pensando en que esa situación nunca va a cambiar, que esa es la vida que eligieron y que así será siempre. El resentimiento que se deriva de este ciclo es una de las consecuencias que más predomina en esta problemática, al estar ligado con un historial de maltrato que ellas han venido soportando al igual que sus hijos. Lo anterior refleja lo señalado por Walker $(1979 ; 1989)$ sobre el ciclo que mantiene la violencia doméstica, por cuanto en los discursos de estas participantes, se observan dos fases de dicho ciclo, la acumulación de tensión y la explosión o agresión, desapareciendo la fase de reconciliación.

Las consecuencias a nivel social están relacionadas con el empobrecimiento y disminución de las redes sociales, ya sea porque se les controla y restringen en forma directa las interacciones con 
familiares, amigos o compañeros de trabajo o porque la vergüenza ante el escarnio público al que son sometidas las lleva a aislarse. Las restricciones que los hombres imponen a sus conyuges así como también la pérdida de la dignidad que experimentan impactan desfavorablemente su autoestima. El maltrato hacia los hijos se convierte también, en muchos casos, en una forma de controlar y someter a la mujer, quienes por evitarlo, renuncian a su derecho a una vida digna. Se encontró que el mayor temor de estas mujeres es a estar solas y desamparadas con hijos pequeños y con pocas probabilidades para ofrecerles un mejor futuro.

Según Fernández et al. (2003), en cuanto al factor social, se puede presentar aislamiento social, pérdida del empleo y ausentismo laboral. También se identificó la pérdida del respeto hacia el papá por parte de los hijos, como lo señala la UNICEF (2000), estas consecuencias no solo tienen relación con la mujer maltratada, sino también tienen efectos secundarios sobre los hijos, ocasionándoles dificultades en el estudio, pérdida del respeto y miedo hacia su padre. En resumen, las consecuencias físicas, psicológicas, sociales y económicas que sufren las mujeres víctimas de violencia afectan su salud mental y física (Glantz et al., 2000; Rincón, 2003). De igual forma, coinciden el síndrome de la mujer maltratada planteado por Walker (1989), cuando, en su mayoría, las mujeres se adaptan a la situación aversiva con desesperanza.

Al realizar un análisis de los antecedentes del maltrato, y específicamente de la subcategoría de patrones de crianza, se encontró que a estas mujeres desde niñas les inculcaron la obligatoriedad de permanecer unidas a sus cónyuges por encima de cualquier circunstancia; de hecho, manifiestan que en sus familias nucleares también existió maltrato y en épocas anteriores era muy normal que la mujer fuera vulnerada por parte de su esposo, lo cual se relaciona con lo mencionado por Jewkes (2002 citado por Fernández et al., 2003), quien señala que una cultura de violencia ayuda a la tolerancia de la misma y a la perpetuación de conflictos. Otros antecedentes del maltrato fueron el consumo de alcohol en los cónyuges, tal como lo señalan Ortega-Ceballos et al. (2007), y las conductas direccionadas a que la mujer no ejerciera ningún trabajo, lo que luego se convertía en una excusa para maltratar a la mujer debido a que en lo económico, ella no aportaba en el hogar (Álvarez $\&$ Hartog, 2005).

Por otra parte, en las mujeres se identifican atribuciones internas sobre el maltrato, ya que eximen de la responsabilidad del acto violento al agresor y asumen "la culpa", expresando que ellas "se lo merecían" o "se lo buscaron por no obedecer o complacer a su marido". Se encuentran atribuciones externas, donde exoneran a sus cónyuges de su responsabilidad frente al acto violento y frente al cambio, al esgrimir que se trata de un rasgo de personalidad, "él es así y no puede cambiar", y que la irascibilidad que presentan, es causa de las agresiones, tal como lo plantean (Álvarez \& Hartog, 2005). Algunas mujeres atribuyen el haber permitido el maltrato, en un primer momento, al "afecto" que sentían por sus parejas y, posteriormente, a que no encontraban una alternativa para salir de la relación abusiva. Según Blando et al. (2004), la violencia hacia la mujer puede aparecer debido a una percepción de indefensión y de inferioridad de las mismas, lo cual genera un desequilibrio al interior de la relación. Según Fernández et al. (2003), como consecuencia de esto, se ha mantenido en el tiempo el rol del hombre como quien tiene el poder y controla, y el de la mujer como aquella debe ser sumisa.

\section{Discusión}

A partir de los relatos de las participantes, se devela un cuadro bastante complejo de la violencia doméstica o de violencia de pareja, tal como lo plantean Vatnar y Bjørkly (2014), O’Doherty, et al. (2014), Lilly y Graham-Bermann (2010), Saftlas et al. (2014), Singh et al. (2014), donde se ponen en tensión las relaciones sujeto-sociedad, ya que la forma en que cada individuo asume su lugar en el mundo, incluyendo la manera de relacionarse con los otros, en este caso siendo ese otro su pareja, está regulada por la cultura, la cual prescribe los guiones de interacción entre los géneros, aún en 
los espacios del llamado "mundo privado". Ello coincide también con lo señalado por Hayati et al. (2013) sobre la tensión y conflicto que experimenta la mujer entre escapar o permanecer en la relación violenta, entre oponerse activamente a la violencia o rendirse a ella y tolerarla. En este sentido, Molina et al. (2010) plantean que la mirada desde la cual se observa el fenómeno de la violencia doméstica trasciende el "simple" acto de la agresión ya que este se enmarca en "un trasfondo ideológico, con formas de ver y pensar el mundo que han sido establecidas socialmente, generación tras generación e impactan el comportamiento de los individuos y sus formas de interacción" (p. 140).

Por otra parte, si se sigue el planteamiento de Foucault (1983) sobre cómo toda interacción humana implica una relación de poder, resulta imprescindible análizar el desarrollo que han tenido los dispositivos de poder y control, los cuales son cada vez más sofisticados y se evidencian en la dinámica de la violencia doméstica. A través del discurso de las participantes, se puede develar como se recrean, en sus relaciones de pareja esos juegos de poder, en donde el hombre asume un rol autoritario e intenta controlar a la mujer, por medio del ejercicio de la violencia, quien a su vez asume un rol pasivo, el cual se va configurando como una espiral patológica que incluye emociones, cogniciones y comportamientos. Así, en el presente estudio se pudo develar cómo la desesperanza, la baja autoestima, el miedo y las atribuciones sobre la violencia doméstica que contribuyen a su normalización y naturalización, se encuentran en la base del andamiaje que la perpetúa y la reproduce.

Si se quiere hacer prevención eficaz de la violencia en cualquiera de sus niveles, resulta imprescindible atender a la propuesta de Molina et al. (2010) de cómo las opciones terapéuticas dirigidas a proporcionarles a las mujeres la ayuda que necesitan, deben estar orientadas a que ellas tengan la "posibilidad de reencontrarse con ellas mismas, de reconciliarse con la vida y de recobrar el valor, que les fue arrebatado fruto de las situaciones vividas con su pareja, a tal punto de experimentar minusvalía e incapacidad para enfrentar el mundo de manera independiente" (p. 146).

\section{Referencias}

Álvarez, J., \& Hartog, G. (2005). Manual de prevención de violencia intrafamiliar. México: Trillas.

Banco Mundial (2002). Informe sobre el desarrollo mundial de 2002. Instituciones para los mercados. Panorama general. Washington: Autor.

Banco Mundial (2012). Informe sobre el desarrollo mundial de 2012. Igualdad de género y desarrollo. Panorama general. Washington: Autor.

Bonilla, E., \& Rodríguez, P. (1995). Más allá del dilema de los métodos. Bogotá: Presencia.

Bosh, E., Ferrer, V., \& Alzamora, A. (2006). El laberinto patriarcal. Reflexiones teórico-prácticas sobre la violencia contra las mujeres. Barcelona: Anthropos.

Blanco, P., Ruiz-Jarabo, C., García de Vinuesa, L., \& Martín-García, M. (2004). La violencia de pareja y la salud de las mujeres. Gaceta Sanitaria, 18(1), 182-188.

Carrasco, C. (2007). Violencia doméstica. Lo que revela la investigación básica con parejas. Papeles del Psicólogo, 28(1), 57-62.

Carey, M. (2003). El efecto del grupo en los grupos focales: planear, ejecutar e interpretar la investigación con grupos focales. En J. Morse (Ed.), Asuntos críticos en los métodos de investigación cualitativa (pp. 262-280). Medellín: Universidad de Antioquia.

Davis, R. E. (2002). "The strongest women”: Exploration of the inner resources of abused women. Qualitative Health Research, 12(9), 1248-1263. doi: 10.1177/1049732302238248

Echeburúa, E., \& De Corral, P. (2009). Manual de violencia intrafamiliar. Madrid: Siglo XXI.

Ellsberg, M. C., \& Heise, L. (2005). Researching violence against women: A practical guide for researchers and activists. Washington: World Health Organization \& Program for Appropriate Technology in Health.

Fernández, M., Herrero, S., Buitrago, F., Ciurana, R., Chocron, L., García, J., ... Tizón, J. L. (2003). Violencia doméstica. Madrid: Ministerio de Sanidad y Consumo.

Flanagan, J., Jaquier, V., Overstreet, N., Swan, S., \& Sullivan, T. (2014). The mediating role of avoidance coping between intimate partner violence (IPV) victimization, mentalhealth, and substance abuse 
among women experiencing bidirectional IPV. Psychiatric Research, 220(1-2), 1-1.

Fondo de Desarrollo de las Naciones Unidas para la Mujer, Fondo de Población de las Naciones Unidas \& Organización Internacional para las Migraciones (2010). Estudio sobre tolerancia social e institucional a la violencia basada en género en Colombia. Programa integral contra violencias de género. Recuperado de: http://www.mdgfund.org/sites/default/ files/GEN_ESTUDIO_Colombia_Tolerancia\%20 social $\% 20 \mathrm{e} \% 20$ institucional $\% 20 \mathrm{a} \% 201 \mathrm{a} \% 20$ violencia\%20de\%20genero.pdf

Fondo Internacional de Emergencia de las Naciones Unidas para la Infancia (2000). La violencia doméstica contra mujeres y niñas. Recuperado de: http:// www.unicef-irc.org/publications/pdf/digest6s.pdf

Foucault, M. (1983). El discurso del poder. México: Folios.

Gage, A. (2005). Women's experience of intimate partner violence in Haiti. Social Science $\mathcal{E}$ Medicine, 61(2), 343-364.

Garrido, V., \& Morales, L. (2003). Psicópatas y otros delincuentes violentos. España: Tirant lo Blanch.

Glantz, N. M., Halperin, D., \& Hunt, L. (2000). Estudiando la violencia doméstica en Chiapas, México. University of Guadalajara Press, 141-160.

Hayati, E. L., Eriksson, M., Hakimi, M., Högberg, U., \& Emmelin, M. (2013). 'Elastic band strategy': Women's lived experiences of coping with domestic violence in rural Indonesia. Global Health Action, 6, 188194. doi: 10.3402/gha.v6i0.18894

Hindin, M., Kishor, S., \& Ansara, D. (2008). Intimate partner violence among couples in 10 DHS countries: Predictors and health outcomes (DHS Analytical Studies No. 18). Calverton, Maryland: Macro International Inc.

Incháustegui, T., \& Olivares, E. (2011). Modelo ecológico para una vida libre de género. México: Comisión Nacional para Prevenir y Erradicar la Violencia contra las Mujeres. Recuperado de: http://cedoc.inmujeres.gob.mx/lgamvlv/MoDecoFinalPDF.pdf

Instituto Nacional de Medicina Legal y Ciencias Forenses (2013). Comportamiento de las lesiones por violencia intrafamiliar. Bogotá: Autor. Recuperado de: http://www.medicinalegal.gov.co/documents/10180/188820/FORENSIS+2013+0+POR
TADA+Y+preliminares.pdf/2ec9f133-6274-441eb928-b09841bec969

Instituto Nacional de Medicina Legal y Ciencias Forenses (2014). Boletín estadístico mensual. Enero 2013. Bogotá: Autor. Recuperado de: http://www.medicinalegal.gov.co/documents/10180/560640/1+enero. pdf/e498ab33-fc55-4af0-91a6-4abf056674ed

Instituto Nacional de Mujeres (2006). Violencia de género en las parejas mexicanas. Análisis de resultados de la Encuesta Nacional sobre la Dinámica de las Relaciones en los Hogares. Componente para mujeres unidas o casadas de 15 años y más. México: Autor. Recuperado de http://cedoc.inmujeres.gob.mx/ documentos_download/100925.pdf

Kleinke, C. (2001). Cómo afrontar los desafíos de la vida. Bilbao: Descleé de Brower.

Krause, E. D., Kaltman, S., Goodman, L. A., \& Dutton, M. A. (2008). Avoidant, coping and PTSD symptoms related to domestic violence exposure: A longitudinal study. Journal of Traumatic Stress, 21(1), 83-90. doi: 10.1002/jts.20288

Labrador, F., Paz, P., De Luis, P., \& Fernández-Velasco, R. (2008). Mujeres víctimas de la violencia doméstica. Madrid: Pirámide.

Labrador, F., Arinero, M., \& Crespo, M. (2004). Efectos psicopatológicos del maltrato doméstico. Avances en Psicología Latinoamericana, 22(1), 105-116.

Lazarus, R. (2000). Estrés y emoción. Manejo e implicaciones en nuestra salud. Bilbao: Descleé de Brouwer.

Lazarus, R. S., \& Folkman, S. (1984). Stress, appraisal and coping. New York: Springer.

Lazarus, R., \& Folkman, S. (1988). Manual for the ways of Coping Questionnaire. Palo Alto, CA: Consulting Psychologist Press.

Lila, M. (2010). Investigación e intervención en violencia contra la mujer en las relaciones de pareja. Intervención Psicosocial, 19(2), 105-108.

Lilly, M. M., \& Graham-Bermann, S. A. (2010). Intimate partner violence and PTSD: The moderating role of emotion-focused coping. Violence and Victims, 25(5), 604-616.

López, C. P., Murad, R., \& Calderón, M. C. (2013). Historias de violencia, roles, prácticas y discursos legitimadores.Violencia contra las mujeres en Colombia 2000-2010. En Encuesta Nacional de Demografía y Salud. ENDS 1990/2010. Serie de Estudios a 
Profundidad. Bogotá: Ministerio de Salud \& Profamilia. Recuperado de http://www. Profamilia.org. co/...7\%20-\%20VI...

Molina, J., Moreno, J., \& Vásquez, H. (2010). Análisis referencial de las representaciones sociales sobre la violencia doméstica. Acta Colombiana de Psicología, 13(2), 129-148.

Murad, R., Gómez, C. P., \& Calderón, M. C. (2013). Historias de violencia, roles, prácticas y discursos legitimadores. Violencia contra las mujeres en Colombia 2000 - 2010. Serie de Estudios a Profundidad. Encuestas Nacionales de Demografía y Salud. ENDS 1990 - 2010. Recuperado de: http:// www. Profamilia.org.co/...7\%20-\%20VI...

O’Doherty, L. J. Taft, A., Hegarty, K., Ramsay, J., Davidson, L. L., \& Feder, G. (2014). Screening women for intimate partner violence in healthcare settings: Abridged Cochrane systematic review and meta-analysis. BMJ, 348, doi: 10.1136/bmj.g2913.

Organización Mundial de la Salud, Escuela de Higiene y Medicina Tropical de Londres \& Consejo Sudafricano de Investigaciones Médicas (2013). Estimaciones mundiales y regionales de la violencia contra la mujer: prevalencia y efectos de la violencia conyugal y de la violencia sexual no conyugal en la salud (Informe OMS 9789241564625). Ginebra: OMS. Recuperado de http://www.who.int/reproductivehealth/publications/violence/9789241564625/es

Organización Panamericana de la Salud (2003). El informe mundial sobre la violencia y la salud (Publicación científica y técnica N. ${ }^{\circ}$ 588). Washington DC: Autor. Recuperado de: http://whqlibdoc.who.int/ publications/2003/9275315884_spa.pdf

Ortega-Ceballos, P. A., Mudgal, J., Flores, Y., RiveraRivera, L., Díaz-Montiel, J. C., \& Salmerón, J. (2007). Determinantes de violencia de pareja en trabajadoras del IMSS, Morelos. Salud Pública de México, 49, 357-366.

Pineles, S. L., Street, A. E., Moustoufi, M., Ready, C. B., Street, A. E., Griffin, M. G., \& Resick, P. A. (2011). Trauma reactivity, avoidant coping, and PTSD symptoms: A moderating relationship? Journal of Abnormal Psychology, 120(1), 240-246.
Rincón, P. (2003). Trastorno de estrés postraumático en mujeres víctimas de violencia doméstica: evaluación de programas de intervención (Tesis doctoral, Universidad Complutense de Madrid, España).

Rodríguez, J. (1995). Psicología social de la salud. Madrid: Síntesis.

Saftlas, A., Harland, K., Wallis, A., Cavanaugh, J., Dickey, P., \& Peek-Asa, C. (2014). Motivational interviewing and intimate partner violence: $\mathrm{A}$ randomized trial. Annals of Epidemiology, 24(2), 144-150.

Sanmartín, J., Molina, A., \& García, Y. (2003). Centro Reina Sofía: violencia contra la mujer en las relaciones de pareja. Madrid: Diseñarte.

Singh, V., Petersen, K., \& Singh, S. R. (2014). Intimate partner violence victimization: Identification and response in primary care. Primary Care: Clinic in Office Practice, 41(2), 261-281. doi: 10.1016/j. pop.2014.02.005

Vatnar, S., \& Bjørkly, S. (2014). An interactional perspective on coping with intimate partner violence: Counterattack, call for help, or give in and obey him? Journal of Aggression, Maltreatment $\mathcal{E}$ Trauma, 23(9), 881-900. doi: 10.1080/10926771.2014.953716

Walker, L. (1979). The battered woman. New York: Harper \& Row Publisher.

Walker, L. (1989). Psychology and violence against women. American Psychologist, 44(4), 695-702.

Walker, L. (1999). Psychology and domestic violence around the world. American Psychologist, 54(1), 21-29.

Walker, L. (2012). El síndrome de la mujer maltratada. Bilbao: Desclée de Brouwer.

World Health Organization. (2005). Multi-country study on women's health and domestic violence against women: Summary report of initial results on prevalence, health outcomes and women's responses. Ginebra: Autor.

Zolotor, A. J., Denham, A. C., \& Weil, A. (2009). Intimate partner violence. Primary Care: Clinic in Office Practice, 36(1), 167-179. doi:10.1016/j. pop.2008.10.010 